International Mathematical Forum, 2, 2007, no. 15, 705 - 711

\title{
Best Approximation in Reflexive Subspaces of $C(Q)$
}

\author{
H. Mazaheri and A. Norouzi \\ Department of Mathematics \\ Yazd University \\ Yazd, Iran \\ hmazaheri@yazduni.ac.ir
}

\begin{abstract}
The purpose of this paper is to introduce and discuss the consept of best approximation for subspaces of $C(Q)$. We shall characterize polynomials of best approximation for given $f \in C(Q)$ in the reflexive subspaces of $C(Q)$. We also show that any commutative $C^{*}$-algebra with identity has not any quasi-Chebyshev maximal ideal.
\end{abstract}

Mathematics Subject Classification: 41A50, 41A52, 46J40, 46J05

Keywords: best approximation, proximinality, Chebyshev sets, quasiChebyshev sets, closed ideals, Klomogorov's type

\section{Introduction and preliminaries}

Let $M$ be a subset of the normed linear space $X$. For any $x_{0} \in X$, the (possibly empty) set of best approximations to $x$ from $M$ is defined by

$$
P_{M}(x)=\{y \in M:\|x-y\|=d(x, M)\},
$$

where $d(x, M)=\inf \{\|x-y\|: y \in M\}$.

The subset $M$ is said to be proximinal (resp., Chebyshev) if $P_{M}(x)$ is nonempty (resp., is a singleton) for each $x \in X$.

A necessary condition for proximinality of a subset $M$ of a normed linear space $X$ is closeness (see, [3]). Also a sufficient condition for this, is compactness (i.e., each sequence of elements of $M$ has a subsequence convergent to an element of $M$ ). Indeed, for each $x \in X$, the functional $e_{x}: M \rightarrow \mathbf{R}$ defined by $e_{x}(m)=\|m-x\|$ is continuous (see, [7, p. 391]) and hence must achieve its minimum on any compact set $M$.

For example every finite-dimensional subspace of a normed linear space or any 
closed ideal of a $C^{*}$-algebra, is proximinal. Also any proximinal subset of a strictly convex space is Chebyshev. (see, [1])

\section{Best approximation in reflexive subspaces of $C(Q)$}

Let $C(Q)$ denotes the space of all continuous, complex valued functions defined on a compact set $Q$ with the supremum norm. In [7] Singer proved Kolomogorov's type for every finite-dimensional subspace $M$ of $C(Q)$. In this section we shall prove kolmogorov's type characterization of best approximation for given $f \in C(Q)$ in a reflexive subspace $M$ of $C(Q)$.

Let $X$ be a normed linear space, $X^{*}$ its dual space and $\mathbf{B}_{X^{*}}$ the closed unit ball in $X^{*}$. A set $E \subseteq \mathbf{B}_{X^{*}}$ is said to be a norming set if

$$
\|x\|=\sup \{|\phi(x)|: \phi \in E\}
$$

for all $x \in X$. It is well known that for each $x$ in $X$,

$$
\|x\|=\sup \left\{|\phi(x)|: \phi \in \mathbf{B}_{X^{*}}\right\}=\sup \left\{|\phi(x)|: \phi \in \operatorname{ext}\left(\mathbf{B}_{X^{*}}\right)\right\}
$$

where, $\operatorname{ext}(S)$, denotes the extreme points of the set $S$.

For a reference we give the following lemma which was introduced by [5].

Lemma 2.1. Let $X$ be a Banach space, $M$ be a reflexive subspace of $X$, $x \in X \backslash M$ and $y_{0} \in M$. Moreover, assume that $E \subseteq B_{X^{*}}$ is an arbitrary norming set. Then $y_{0}$ belongs to $P_{M}(x)$ if and only if for every $\varepsilon>0$ there exist $\phi_{1}, \phi_{2}, \cdots, \phi_{n} \in E$ and $\lambda_{1}, \lambda_{2}, \cdots, \lambda_{n} \in \boldsymbol{C}$ such that $\sum_{i=1}^{n}\left|\lambda_{i}\right| \leq 1$,

$$
\left|\sum_{i=1}^{n} \lambda_{i} \phi_{i}(y)\right|<\varepsilon\|y\|
$$

for all $y \in M$, and

$$
\left|\left\|x-y_{0}\right\|-\sum_{i=1}^{n} \lambda_{i} \phi_{i}\left(x-y_{0}\right)\right|<\varepsilon
$$

Theorem 2.2. Let $M$ be a reflexive subspace of $C(Q)$. Assume that $f \in$ $C(Q) \backslash M$ and $g_{0} \in M$. Then $g_{0} \in P_{M}(f)$ if and only if for every $\varepsilon>0$, there exist $q_{1}, q_{2}, \cdots, q_{n} \in Q$ and $\lambda_{1}, \lambda_{2}, \cdots, \lambda_{n} \in C$ such that $\sum_{i=1}^{n}\left|\lambda_{i}\right| \leq 1$,

$$
\left|\sum_{i=1}^{n} \lambda_{i} g\left(q_{i}\right)\right|<\varepsilon\|g\|
$$


for all $g \in M$, and

$$
\left|\left\|f-g_{0}\right\|-\sum_{i=1}^{n} \lambda_{i}\left(f-g_{0}\right)\left(q_{i}\right)\right|<\varepsilon .
$$

Proof. We set $E=\operatorname{ext}\left(B_{X^{*}}\right)$ (where, $X=C(Q)$ ). By the fact mentioned just before the Lemma 2.1, it is clear, that $E$ is a norming set in $B_{X^{*}}$. By Lemma 2.1, hence, $g_{0} \in P_{M}(f)$ if and only if for every $\varepsilon>0$ there exist $\phi_{1}, \phi_{2}, \cdots, \phi_{n} \in E$ and $\alpha_{1}, \alpha_{2}, \cdots, \alpha_{n} \in \mathbf{C}$, such that $\sum_{i=1}^{n}\left|\alpha_{i}\right| \leq 1$,

$$
\left|\sum_{i=1}^{n} \alpha_{i} \phi_{i}(g)\right|<\varepsilon\|g\|
$$

for all $g \in M$, and

$$
\left|\left\|f-g_{0}\right\|-\sum_{i=1}^{n} \alpha_{i} \phi_{i}\left(f-g_{0}\right)\right|<\varepsilon .
$$

It is known that $f \in X^{*}$ is an extremal point of $B_{X^{*}}$ if and if there exists an element $q \in Q$, and a scalar $\beta \in \mathbf{C}$ with $|\beta|=1$, such that

$$
f(y)=\beta y(q) \quad(y \in C(Q)) .
$$

For each $1 \leq i \leq n, \phi_{i} \in \operatorname{ext}\left(B_{X^{*}}\right)$, therefore there exists a $q_{i}$ in $Q$ and a scalar $\beta_{i}$ with $\left|\beta_{i}\right|=1$, such that

$$
\phi_{i}(g)=\beta_{i} g\left(q_{i}\right) \quad(y \in C(Q)) .
$$

Putting $\lambda_{i}=\alpha_{i} \beta_{i}$, then, we have

$$
\begin{aligned}
\left|\sum_{i=1}^{n} \lambda_{i} g\left(q_{i}\right)\right| & =\left|\sum_{i=1}^{n} \alpha_{i} \beta_{i} g\left(q_{i}\right)\right| \\
& =\left|\sum_{i=1}^{n} \alpha_{i} \phi_{i}(g)\right|<\varepsilon\|g\|
\end{aligned}
$$

for all $g \in M$, and

$$
\left|\left\|f-g_{0}\right\|-\sum_{i=1}^{n} \lambda_{i}\left(f-g_{0}\right)\left(q_{i}\right)\right|=\left|\left\|f-g_{0}\right\|-\sum_{i=1}^{n} \alpha_{i} \phi_{i}\left(f-g_{0}\right)\right|<\varepsilon .
$$

The following corollary is an immediate consequence of Theorem 2.2 and the next one (Corollary 2.4) is a consequence of a result from [5] and Corollary 2.2 . 
Corollary 2.3. Let $M$ be a reflexive subspace of $C(Q)$. Assume that $f \in$ $C(Q) \backslash M$ and $F \subseteq M$. Then $F \subseteq P_{M}(f)$ if and only if for every $\varepsilon>0$ there exist $q_{1}, q_{2}, \cdots, q_{n} \in Q$ and $\lambda_{1}, \lambda_{2}, \cdots, \lambda_{n} \in \boldsymbol{C}$ such that $\sum_{i=1}^{n}\left|\lambda_{i}\right| \leq 1$,

$$
\left|\sum_{i=1}^{n} \lambda_{i} g\left(q_{i}\right)\right|<\varepsilon\|g\|
$$

for all $g \in M$, and

$$
\left|\left\|f-g_{0}\right\|-\sum_{i=1}^{n} \lambda_{i}(f-g)\left(q_{i}\right)\right|<\varepsilon
$$

for all $g \in F$.

Corollary 2.4. Let $M$ be a reflexive subspace of $C(Q)$. Then

(a) $M$ is a non-Chebyshev subspace of $X$.

(b) for every $\varepsilon>0$ there exist $q_{1}, q_{2}, \cdots, q_{n} \in Q$ and $\lambda_{1}, \lambda_{2}, \cdots, \lambda_{n} \in C$ such that $\sum_{i=1}^{n}\left|\lambda_{i}\right| \leq 1$,

$$
\left|\sum_{i=1}^{n} \lambda_{i} g\left(q_{i}\right)\right|<\varepsilon\|g\|
$$

for all $g \in M$, and

$$
\left|\left\|f-g_{0}\right\|-\sum_{i=1}^{n} \lambda_{i}\left(f-g_{0}\right)\left(q_{i}\right)\right|<\varepsilon .
$$

and

$$
\left|\|f\|-\sum_{i=1}^{n} \lambda_{i} f\left(q_{i}\right)\right|<\varepsilon .
$$

\section{$3 \quad$ Best approximation by ideals of $C(Q)$}

To each closed ideal $I$ of $C(Q)$ there is associated a closed subset $F$ of $Q$ such that

$$
I=\{f \in C(Q): f(x)=0 \text { for all } x \text { in } F\}
$$

(see [2]).

To show that the closed subset $F$ of $Q$ is associated to the ideal $I$ of $C(Q)$, we use the expression "the closed ideal $(I, F)$ of $C(Q)$ " rather than "the closed ideal $I$ of $C(Q)$ ".

Lemma 3.1. Let $(I, F)$ be a closed ideal of $C(Q)$, then

(a) $F=\emptyset$ if and only if $I=C(Q)$.

(b) $F=Q$ if and only if $I=\{0\}$.

(c) $F$ is a singleton if and only if $I$ is a maximal ideal of $C(Q)$. 
(d) If $F$ is a finite set then $I$ is a finite codimensional ideal of $C(Q)$.

proof. (a) Given $g \in C(Q)$. Since $F=\emptyset$, for each $x \in Q$, there exists $f_{x} \in I$ such that $f_{x}(x) \neq 0$. By continuity of $f_{x}$, therefore, there is an open set $U_{x}$ containing $x$ such that $f_{x}(t) \neq 0$ for all $t \in U_{x}$. Compactness of $Q$, now implies, the existence of a finite number of elements $x_{1}, x_{2}, \cdots, x_{n}$ in $Q$ such that for each $1 \leq i \leq n, f_{x_{i}}(t) \neq 0$ for all $t \in U_{x_{i}}$. Putting $f=f_{x_{1}}^{2}+f_{x_{2}}^{2} \cdots+f_{x_{n}}^{2}$ it follows that $f(x) \neq 0$ for all $x \in C(Q)$. Since $f \in I$ and $I$ is an ideal, $g=f \cdot\left(\frac{g}{f}\right) \in I$. Therefore $I=C(Q)$.

The implication " $I=C(Q) \Rightarrow F=\emptyset "$ and parts (b) and (c) are trivial. If $F=\left\{x_{1}, x_{2}, \cdots, x_{n}\right\} \subseteq Q$, for $i=1,2, \cdots, n$, define

$$
\Lambda_{i}(f)=f\left(x_{i}\right) \quad(f \in C(Q) .
$$

Then, for each $1 \leq i \leq n, \Lambda_{i}$ is a linear functional on $C(Q)$. Since

$$
I=\left\{f \in C(Q): \Lambda_{i}(f)=0, \quad \text { for all } i=1,2, \cdots, n\right\}
$$

it follows that $I$ is of finite codimension in $C(Q)$ which completes the proof.

Following notations of section 1 , the set $M$ is called a quasi-Chebyshev subset of $X$ if $P_{M}(x)$ is a nonempty compact subset of $M$, for all $x$ in $X$.

For example any nonempty closed subspace of a finite dimensional space $X$ is quasi-Chebyshev. (see, [4])

We call a nonzero ideal $I$ of $C(Q)$, which is not $C(Q)$, a nontrivial ideal of $C(Q)$.

Theorem 3.2. Let $(I, F)$ be a closed nontrivial ideal of $C(Q)$ such that $\operatorname{int}(I)=\emptyset$. Then $I$ is not a quasi-Chebyshev ideal of $C(Q)$.

proof. There exists $x_{0} \in F$ such that $g\left(x_{0}\right)=0$, for all $g \in I$. Choose $f \in C(Q)$ such that $f\left(x_{0}\right)=1$ and $0 \leq f \leq 1$. We want to show that $P_{I}(f)$ is not compact in $I$. By Ascoli's Theorem (see, for example, [6, p. 277]), it suffices to show that $P_{I}(f)$ is not equicontinuous at $x_{0}$. To do this end, we must find an $\varepsilon>0$ such that for every neighborhood $U$ of $x_{0}$ there exist $x_{1} \in U_{x}$ and $h \in P_{M}(f)$ such that $\left|h\left(x_{0}\right)-h\left(x_{1}\right)\right| \geq \varepsilon$.

Put $\varepsilon=1 / 2$, and let $U$ be a neighborhood of $x_{0}$. Since $U$ is open and $\operatorname{int}(F)=\emptyset, U$ is not a subset of $F$ and hence there exists an element $x_{1}$ in $U$ which is not in $F$. Since $x$ is not in $F$, there exists $g \in I$ such that $g\left(x_{1}\right) \neq 0$. Define

$$
h(x)=\frac{g^{2}(x)}{\max \left\{g^{2}(x), g^{2}\left(x_{1}\right)\right\}} \quad(x \in Q)
$$

Then $h$ belongs to $I, 0 \neq h$ and $0 \leq h \leq 1$. Since also $0 \leq f \leq 1,\|f-h\| \leq 1$. On the other hand $\left|f\left(x_{0}\right)-h\left(x_{0}\right)\right|=1$, so that $\|f-h\|=1$. 
For all $g \in I$, however, $\|f-g\| \geq\left|f\left(x_{0}\right)-g\left(x_{0}\right)\right|=1$. Since $h$ belongs to $I$ and $\|f-h\|=1$, it follows that $h \in P_{M}(f)$. However, $\left|h\left(x_{0}\right)-h\left(x_{1}\right)\right|=1 \geq \varepsilon$.

Theorem 3.2 in combination with Lemma 3.1 gives the following corollary.

Corollary 3.3. No maximal ideal of $C(Q)$ is a quasi-Chebyshev ideal.

Let $A$ be a commutative $C^{*}$-algebra with identity and let $\Sigma$ be its maximal ideal space. Then the Gelfand transform $\varphi: A \rightarrow C(\Sigma)$ is an isometric *isomorphism of $A$ onto $C(\Sigma)$ (see, e. g., [2, p. 236]).

We use here, the Greek letter $\varphi$ for the Gelfand transform on a $C^{*}$-algebra. The following Lemma is an straightforward consequence of the facts that the Gelfand transform is both onto and isometric.

Lemma 3.4. Let $A$ be a commutative $C^{*}$-algebra then,

(a) $I$ is a maximal ideal of $A$ if and only if $\varphi(I)$ is a maximal ideal of $C(\Sigma)$.

(b) $I$ is a quasi-Chebyshev ideal of $A$ if and only if $\varphi(I)$ is a quasi-Chebyshev ideal of $C(\Sigma)$.

Corollary 3.5. No $C^{*}$-algebra with identity has a quasi-Chebyshev maximal ideal.

proof. Let $A$ be a commutative $C^{*}$-algebra with identity, and let $I$ be a maximal ideal of $A$ which is quasi-Chebyshev. Then from Lemma 3.4, $\varphi(I)$ is a quasi-Chebyshev maximal ideal of $C(\Sigma)$, which contradicts Corollary 3.3.

\section{References}

1. A. S. Akemann, G. K. Pedersen, J. Tomiyama, Multipliers of $C^{*}$-algebra, J. Funct. Anal. 13 (1973), 277-301.

2. J.B. Conway, A Coures in Functional Analysis, Springer-Verlag, (1985).

3. F. Girosi, T. Poggio, Networks and the Best Approximation Property, Biological Cybernetics, 63 (1990), 169-176.

4. H. Mohebi, On quasi-Chebyshev subspaces of Banach spaces, J. Approx. Theory 107 (2000), 87-95. 
5. H. Mohebi, H. Mazaheri, Best approximation in reflexive subpace of $L(X, Y)$, Soochow J. 27 (2001), no. 3, 225-226.

6. J. R. Munkres, Topology A First Course, Prentice-Hall, New Jersey, (1975).

7. I. Singer, Best Approximation in Normad Linear Spaces by Elements of Linear Subspaces, Springer-Verlag (1970).

Received: March 4, 2006 